\title{
Essais
}

Revue interdisciplinaire d'Humanités

\section{Lieux historiques, musées et mémoriaux}

Avant-propos

Hélène Camarade

\section{(2) OpenEdition}

Journals

Édition électronique

URL : http://journals.openedition.org/essais/5541

DOI : $10.4000 /$ essais. 5541

ISSN : 2276-0970

Éditeur

École doctorale Montaigne Humanités

\section{Édition imprimée}

Date de publication : 15 janvier 2015

Pagination : 8-16

ISBN : 978-2-9544269-4-5

ISSN : 2417-4211

Référence électronique

Hélène Camarade, "Lieux historiques, musées et mémoriaux », Essais [En ligne], 6 | 2015, mis en ligne le 30 novembre 2020, consulté le 02 décembre 2020. URL : http://journals.openedition.org/essais/ 5541 ; DOI : https://doi.org/10.4000/essais.5541 


\section{Lieux historiques, musées \\ et mémoriaux}

\section{Avant-propos}

Hélène Camarade

Les lieux, au sens géographique du terme, sont autant de traces du passé. Qu'il s'agisse de sites ou de lieux historiques, parfois transformés en musées ou en lieux d'exposition, qu'il s'agisse de monuments ou de mémoriaux, ou encore de lieux de commémoration, ces espaces nous renseignent sur ce qu'une société montre, raconte, conserve ou évacue de son histoire à un moment donné. Dès lors, observer ces lieux, étudier leur conception, leur agencement ou les transformations qu'ils ont subies au cours du temps, permet de formuler des hypothèses sur la façon dont on écrit l'histoire dans un espace culturel donné.

Ce volume thématique sur "L'histoire par les lieux »" est le fruit d'un projet qui s'est déroulé en plusieurs étapes. Celui-ci a commencé dans le cadre d'un séminaire de formation-recherche qui a eu lieu à Berlin en mars 2012 sur le thème de la concurrence des mémoires ${ }^{2}$. La visite de musées et d'expositions consacrés à l'histoire allemande a sensibilisé les participants aux différentes façons de présenter, raconter ou illustrer l'histoire. Ils ont pu observer combien les réalisations muséographiques, et les objectifs qu'elles présupposent, sont variables en fonction des auteurs et des époques où elles ont été conçues. Ils ont été attentifs aux choix esthétiques qui ont présidé à la construction de monuments et de mémoriaux, par exemple l'impressionnant Mémorial aux Juifs assassinés d'Europe, constitué d'un champ de stèles qui s'étend sur $19000 \mathrm{~m}^{2}$, et le Mémorial aux homosexuels persécutés pendant le national-socialisme, plus discret, qui lui fait face ${ }^{3}$. Ils ont saisi la dimension politique et identitaire d'un

1 Ce titre revient à Hugo Remark, doctorant en études germaniques.

2 Ce séminaire franco-germano-polonais, auquel des étudiants germanistes de l'Université Bordeaux Montaigne, membres de l'EA CLARE, ont participé, a été organisé en collaboration avec la Fondation Friedrich Ebert (et le professeur Friedhelm Boll) et le Volksbund Deutsche Kriegsgräberfürsorge. Les résultats ont été publiés dans la brochure : Volksbund Forum (éd.), Zur Konkurrenz der Erinnerungskulturen in Deutschland, Frankreich und Polen, Kassel, Volksbund Deutsche Kriegsgräberfürsorge e. V., 2012.

3 Cf. http://www.stiftung-denkmal.de (consulté le 12/09/2014). 
mémorial, ainsi que le rôle qu'un pan du passé peut jouer dans le présent pour un groupe social ou toute une société. Ils ont également mesuré le phénomène de stratification de la mémoire, particulièrement saisissant dans la capitale allemande où de nombreux lieux gardent la trace de différentes époques, et notamment des deux dictatures qui se sont succédé au XXe siècle. Un exemple emblématique en est le camp de concentration de Sachsenhausen qui fut utilisé, dès le mois de mai 1945 et jusqu'en 1950, comme camp spécial d'internement et de répression (Speziallager) par la puissance d'occupation soviétique. Ils ont compris qu'une telle sédimentation de la mémoire offre de multiples opportunités d'oubli. Les participants français et polonais ont d'ailleurs su trouver des exemples similaires dans leurs histoires nationales ; en France par exemple avec les camps d'internement de Gurs ou de Rivesaltes réutilisés dès 1944-1945 comme camps de prisonniers allemands. Ils ont enfin été sensibilisés à la gestion bi- ou multinationale des lieux historiques, un fait assez fréquent en Allemagne en raison des déplacements de frontières, par exemple manifeste dans l'existence de lieux de mémoire dits "partagés ${ }^{4}$ avec les pays limitrophes. Ce partage de la mémoire n'est d'ailleurs pas toujours exempt de concurrence entre les mémoires nationales concernées. Le Centre international de rencontres pour la jeunesse de Kreisau, fondé après 1989 sur le domaine de Krzyżowa/Kreisau - lieu qui abrita les activités d'un groupe de résistants allemands dans les années 1940 -, offre aujourd'hui un exemple réussi de mémoire et de lieu partagés, le site se situant en basse-Silésie, un territoire allemand jusqu'en 1945, devenu polonais après la guerre 5 .

L'impulsion créée par le séjour à Berlin a conduit à l'organisation d'un séminaire interdisciplinaire doctoral en janvier 2013 à l'Université Bordeaux Montaigne, lors duquel les organisateurs ont cherché à élargir le champ des réflexions ${ }^{6}$. Ils ont tout d'abord veillé à ouvrir la réflexion à d'autres aires culturelles, notamment à la France, à la Grande-Bretagne et à l'espace européen. Ils ont ensuite convié des chercheurs et doctorants issus de différentes disciplines afin de croiser les problématiques et les approches méthodologiques. Dans

4 „Einleitung“, in Étienne François, Hagen Schulze (éd.), Deutsche Erinnerungsorte, Berlin, Beck, 2001, p. 19. Des ouvrages ont par exemple répertorié des lieux de mémoire germanopolonais ou franco-allemands. Cf. Hans Henning Hahn, Robert Traba (éd.), Deutsch-polnische Erinnerungsorte. Parallelen, Paderborn, Schöningh, 2011 ; Thomas Keller, Jean-Marie Guillon (éd.), "Lieux de migrations/lieux de mémoire franco-allemands ", numéro thématique, in Cahiers d'études germaniques, $\mathrm{n}^{\circ}$ 53, 2007/2.

5 Cf. Hélène Camarade, «Krzyżowa/Kreisau, paradigme du renouveau démocratique et de l'entente européenne : les enjeux de la mémoire germano-polonaise du Cercle de Kreisau depuis 1945 ", in Cultures d'Europe centrale, hors-série n 6, 2009, p. 205-220.

6 Le séminaire "L'histoire par les lieux. Une approche interdisciplinaire des espaces dédiées à la mémoire " a été co-organisé par Hélène Camarade et Hugo Remark, doctorant en études germaniques, avec le soutien de l'École Doctorale Montaigne Humanités et de l'EA CLARE. 
ce numéro thématique, qui résulte en grande partie du séminaire interdisciplinaire doctoral, la sociologie et l'ethnographie sont représentées respectivement par Patrick Baudry et Camille Mazé, la didactique de l'histoire - une discipline à part entière dans l'espace scientifique allemand - par l'historienne Christine Pflüger, les études visuelles par Raphaële Bertho, la narratologie par Hugo Remark, et les civilisations allemande et britannique respectivement par Cécile Denis et Madeline Zielinski. Cette approche plurielle de la thématique a permis de mesurer combien la mémoire que l'on construit ou que l'on efface dans les lieux se trouve à la croisée de la politique, de la didactique, de la culture et des arts.

La réflexion a porté sur des lieux géographiques bien palpables, et non sur les "lieux de mémoire " au sens où ils ont été définis par Pierre Nora dans leur dimension parfois immatérielle et symbolique ${ }^{7}$. Christine Pflüger les qualifie de « lieux historiques » en s'appuyant sur la définition qu'en donne l'historien Ulrich Mayer comme lieux ou cadres d'événements historiques. Ce volume traite cependant surtout de musées et d'expositions permanentes situés souvent, mais pas systématiquement, sur des lieux historiques. Les musées sont des objets d'étude particulièrement intéressants car ils sont à la fois des produits et des vecteurs de la mémoire. Qu'on les considère comme des " arènes " où l'on se bat au nom d'interprétations historiques, ou comme des espaces dans lesquels une politique de la mémoire «se met en scène » afin d'afficher une volonté politique - que l'on pense au projet de la Maison de l'histoire de France voulue par Nicolas Sarkozy ou à la polémique entourant l'ouverture du Musée du 11 septembre à New-York -, les musées sont des sismographes du rapport à l'identité collective et au passé. Dans ce volume, de nombreux musées et mémoriaux sont ainsi passés au crible. Camille Mazé s'intéresse à l'émergence de musées dits de l'Europe après l'ère des musées nationaux. Dans des approches transversales, Christine Pflüger et Hugo Remark portent principalement leur attention sur des musées allemands consacrés à la Seconde Guerre mondiale, alors que Cécile Denis se focalise sur la construction récente d'un musée-mémorial en France, sur le site du camp d'internement Les Milles. Madeline Zielinski s'intéresse pour sa part à la construction tardive d'un lieu de commémoration en Grande-Bretagne, le Mémorial en

7 Pierre Nora, « Entre Mémoire et Histoire. La problématique des lieux », in P. Nora (éd.), Les Lieux de mémoire, vol. 1, Paris, 1984, p. XXXIV.

8 Ulrich Mayer, «Historische Orte als Lernorte », in U. Mayer, H.-J. Pandel, G. Schneider (éd.), Handbuch Methoden im Geschichtsunterricht, Schwalbach/Ts., Wochenschau Verlag, 2004, p. 390 .

9 Joachim Baur, «Museum - Bühne - Arena. Geschichtspolitik und Musealisierung im Zeichen von Globalisierung und gesellschaftlicher Pluralisierung ", in E. François, K. Konczal, R. Traba, S. Troebst (éd.), Geschichtspolitik in Europa seit 1989, Göttingen, Wallstein, 2013, p. 451. 
hommage aux aviateurs du Bomber Command. Deux contributeurs ont poussé la thématique des lieux historiques à ses marges, pourrait-on dire, afin de l'enrichir. Raphaële Bertho s'intéresse à la ville de Dresde dans sa totalité en étudiant la façon dont la représentation picturale de la ville au XVIII ${ }^{\mathrm{e}}$ siècle a servi de modèle pour sa reconstruction après la réunification allemande. Patrick Baudry se consacre à un lieu qui se trouve à la croisée de l'histoire familiale, de l'histoire des sociétés et de celle des territoires, le cimetière. Ce volume analyse ainsi une multitude de lieux inscrits dans l'histoire qui contribuent, à leur tour, à écrire l'histoire.

Avant de croiser quelques résultats de recherche, nous proposons une présentation succincte des différentes problématiques et méthodes utilisées. Le volume s'ouvre sur trois contributions transversales qui fournissent des éclairages spécifiques. Christine Pflüger analyse la question des lieux historiques dans l'espace germanique sous l'angle de la pédagogie et de la didactique, puis dans ses implications politiques, sociales et culturelles. Sa contribution nous rappelle la spécificité que l'apprentissage de l'histoire revêt dans le milieu scolaire allemand puisqu'il reste indissociable d'impératifs civiques et éthiques et qu'il a par exemple conduit à l'élaboration de pratiques pédagogiques liées à l'apprentissage par le vécu et les émotions sur les lieux historiques mêmes.

En s'appuyant sur une enquête de terrain sociohistorique et ethnographique menée entre 2005 et 2010, Camille Mazé analyse une dizaine de projets visant à concevoir des musées de l'Europe dans l'espace européen dans le but de promouvoir l'identité européenne. Elle en étudie les objectifs et les entraves et constate que ce sont surtout les États constructeurs de l'Union européenne, situés en Europe de l'Ouest, qui en sont les moteurs, alors que l'orientation muséographique reste de nature essentiellement nationale en Europe de l'Est.

Considérant les espaces muséographiques comme des " constructions narratives ", Hugo Remark cherche à en analyser le fonctionnement à l'aide d'outils narratologiques. Il utilise le modèle génétique idéal des niveaux narratifs de Wolf Schmid afin de saisir la genèse et la production du récit qui est à l'œuvre dans des expositions permanentes. Ce modèle lui permet également d'étudier la réception des expositions par le visiteur et de mettre au jour le processus d'inférence qui conduit ce dernier à « reconstruire » le passé évoqué. Il analyse et compare également différents discours-cadres censés accompagner et contextualiser la visite.

Les deux contributions suivantes sont des études de cas, l'un français, l'autre britannique, qui étudient dans des perspectives diachroniques la résurgence de pans jusqu'ici absents des mémoires nationales, l'un parce qu'il a été oublié, l'autre parce qu'il est resté controversé. Cécile Denis retrace la périodisation de la mémoire du camp d'internement Les Milles en l'inscri- 
vant dans celle de la mémoire française de Vichy et celle de la mémoire des exilés allemands en France. Elle s'interroge sur les entraves de ce « retour de la mémoire " et sur ceux qui en ont été les moteurs. Elle fait ensuite une analyse du parcours muséographique en trois temps proposé dans le musée-mémorial qui a ouvert ses portes en 2012.

Madeline Zielinski adopte une démarche similaire en analysant tout d'abord les causes de l'oubli délibéré dans lequel sont tombés les aviateurs du Bomber Command, ce commando britannique chargé pendant la Seconde Guerre mondiale de bombarder l'Allemagne, puis celles de leur réhabilitation partielle à partir des années 1990, notamment dans le contexte des différentes guerres auxquelles la Grande-Bretagne a participé, qui mène à la construction en 2012 d'un Mémorial en leur honneur. Ces deux contributions, axées sur les aléas des mémoires nationales, mettent en perspective la communication de Camille Mazé consacrée aux difficultés rencontrées pour faire émerger une mémoire européenne.

Les deux communications qui viennent clore le numéro thématique élargissent l'angle de réflexion en faisant intervenir des éléments nouveaux, notamment la question de l'espace urbain. Raphaële Bertho étudie la façon dont la reconstruction de la ville de Dresde, détruite pendant la Seconde Guerre mondiale, a été poursuivie, après la réunification allemande, à partir d'une représentation de la ville fantasmée par la peinture, et notamment par les vedute de Bernardo Bellotto, conduisant à la construction - plutôt qu'à la reconstruction - d'un Dresde qui n'a finalement jamais existé. L'auteur analyse le processus mémoriel qui conduit, de 1945 à nos jours, à l'élaboration de cet imaginaire urbain qui n'a que peu de rapport avec le passé architectural de la ville. On savait déjà que la fiction cinématographique ou romanesque pouvait prendre le pas sur l'archive dans la construction mémorielle ; l'auteur montre que c'est également le cas de la "fiction picturale".

À partir d'une réflexion sur la place que nos espaces urbains accordent aujourd'hui aux cimetières, Patrick Baudry aborde la question de celle des morts dans nos sociétés. En mettant cette thématique en perspective, historiquement et par le biais de la comparaison aux sociétés non occidentales, il explique comment le tissu urbain, après avoir évacué le cimetière à sa périphérie afin de le rendre " invisible ", cherche désormais à l' " absorber " dans une logique de " désymbolisation ». L'auteur interprète l'abandon de la ritualité et des élaborations qu'elle implique - séparation d'avec le défunt, remaniement de la relation avec lui et réorganisation des " places dans la chaîne générationnelle »-comme le refus de se confronter à ce que la mort implique, à savoir l'inconnu et l'altérité, au profit d'une ritualisation qui privilégie la relation entre les vivants.

Plusieurs fils conducteurs se dégagent des communications. Un premier point commun à la plupart d'entre elles réside dans l'attention portée au phénomène de périodisation de la mémoire. La démarche diachronique, souvent 
adoptée, permet de mettre au jour les évolutions et transformations que les lieux ont subies, et d'ainsi contribuer à écrire une " histoire de la mémoire ${ }^{10}$. Cette dimension temporelle est également sous-jacente dans les contributions non diachroniques. En comparant le discours-cadre d'une exposition permanente élaborée au cours des années 1980 à celui d'une exposition récente, Hugo Remark constate par exemple que le discours muséal de cette dernière intègre un discours autoréflexif sur l'écriture de l'histoire, absent de la première. L'évolution muséographique a ici logiquement tenu compte des réflexions qu'ont menées les historiens au cours des trente dernières années sur la relativité de la connaissance en histoire et sur le conflit des interprétations. Les différentes contributions permettent d'ailleurs de mesurer que les années 1980, puis surtout les années 1990, ont joué un rôle décisif dans l'évolution muséographique. C'est à cette date que l'on assiste, selon Camille Mazé, aux premières tentatives afin d'européaniser les musées d'histoire (le Deutsches historisches Museum à Berlin et l'Historial de la Grande Guerre de Péronne en France par exemple). Cécile Denis montre que c'est également une période-clé dans les projets autour du camp Les Milles. Plusieurs facteurs peuvent contribuer à expliquer ce tournant. La fin des années 1980 et les années 1990 correspondent au moment où la mémoire devient, dans le contexte des réflexions menées sur la relativité de la connaissance historique, un sujet d'études à part entière, du moins en France et en Allemagne : on débat alors sur le rôle des témoins et de l'histoire orale dans l'écriture de l'histoire, Pierre Nora mène ses réflexions critiques sur les lieux de mémoire, on assiste à un retour sur la mémoire de Vichy en France et à la querelle des historiens en Allemagne, etc. ${ }^{11}$ Ces années coïncident bien sûr aussi avec la fin de la Guerre froide qui ouvre la voie à des interprétations historiques moins partisanes, ou à des conceptions plus apaisées, voire réconciliées, de faits historiques.

Les auteurs de ce numéro s'interrogent également sur les acteurs de la mémoire, sur ceux par qui la mémoire évolue ou les thèmes oubliés resurgissent. Plusieurs contributions permettent ainsi de mesurer combien les évolutions sont souvent le fruit de confrontations, parfois conflictuelles, entre la société civile, la recherche historique et les pouvoirs publics. En GrandeBretagne, c'est aux termes de vifs débats historiques qu'une initiative portée par les médias et soutenue - voire promue - par la classe politique et la famille royale conduit au projet de Mémorial aux aviateurs du Bomber Command, comme le montre Madeline Zielinki. Les tournants que l'on constate dans

10 Henry Rousso, "Les dilemmes de la mémoire européenne ", in Christian Delacroix, François Dosse, Patrick Garcia (éd.), Historicités, Paris, La Découverte, 2009, p. 207 (première publication : "Das Dilemna eines europäischen Gedächtnis », in Zeithistorische Forschungen, Nr. 1, 3/2004).

11 On pense ici aux travaux de Pierre Nora et Henry Rousso en France ou Norbert Frei en Allemagne. 
l'histoire des lieux coïncident par ailleurs souvent avec la diversification ou le renouvellement des personnes les animant. Christine Pflüger note ainsi que les musées d'histoire sont désormais conçus en Allemagne par des muséologues, des historiens mais aussi des enseignants et des pédagogues. Cécile Denis souligne le caractère pluridisciplinaire du conseil scientifique de la Fondation du camp Les Milles qui gère désormais le Mémorial et qui a permis de dépasser la concurrence des mémoires de ce camp.

Un autre point commun renvoie à l'analyse de la scénographie mise en place dans certains musées et expositions. L'organisation de l'espace fait en effet souvent partie intégrante du concept muséographique, comme au site-mémorial du camp Les Milles divisé en trois espaces distincts, le musée d'histoire qui livre des informations historiques, le lieu historique (en l'occurrence les salles conservées du camp) qui fait appel aux émotions des visiteurs, et enfin le musée d'éducation civique et citoyenne qui invite ces derniers à tirer du passé des enseignements pour le présent - ces trois espaces se succédant au fil de la même visite. Christine Pflüger souligne elle aussi que la collaboration entre diverses professions liées à la mémoire (muséographe, historien et pédagogue) a conduit à ce que l'on délimite désormais plus nettement les espaces par leurs fonctions, lieux de commémorations d'une part et parcours muséographique de l'autre. En déambulant dans les lieux historiques, dans la salle du réfectoire au camp Les Milles ou dans les allées du camp de Sachsenhausen par exemple, les visiteurs sont censés faire ce que Christine Pflüger nomme «l'expérience du réel ", c'est-à-dire prendre conscience que ces lieux ont bien été des cadres de vie et que les événements historiques, pour inconcevables qu'ils puissent paraître parfois, ont bel et bien eu lieu. C'est en vertu de « l'effet de réel $»^{12}$ qu'ils produisent que l'on conserve si précieusement - ou que l'on restaure les graffitis inscrits sur les murs par les détenus. Hugo Remark parvient à une conclusion analogue lorsqu'il s'interroge sur le rôle assigné aux objets traditionnellement exposés dans les musées. Il démontre que ceux-ci sont souvent conçus comme les "supports métonymiques d'un récit muet " qui permettent au visiteur " par effet de réel » de reconstituer des morceaux du passé évoqué. Montrer les sabots portés par les détenus des camps permettrait au visiteur de se représenter, presque dans sa chair, leur vie quotidienne. L'organisation des espaces mémoriels chercherait ainsi à rappeler que les événements évoqués ont eu lieu dans le monde réel. Dès lors, il est troublant de lire sous la plume de Raphaële Bertho que c'est justement en vertu de « l'illusion de réalisme » qu'ils dégagent que les vedute de Belloto, que l'on compare souvent à des prises de vue photographiques, se sont si facilement substitués à des archives picturales de la ville de Dresde. L'« effet de réel », qui n'est donc bien ici qu'un effet au sens barthien du terme, se retourne contre l'adéquation à l'histoire architecturale

12 Roland Barthes, "L'effet de réel ", in Communications, n 11, 1968, p. 84-89. 
de la ville. Dans le cas de Dresde, on peut parler d'une scénographie à l'échelle de tout le centre-ville puisque l'on assiste, pendant la phase de reconstruction, à la création d'un véritable " décor " constitué de reproductions de Belloto, elles-mêmes d'ailleurs remaniées, qui sont tendues à l'aide de bâches sur les façades des bâtiments. C'est ici la création d'un vrai décor de carton-pâte. La question de la scénographie a également mené certains auteurs à s'intéresser aux jeux de lumière ou à la présence de sons et de musiques dans les musées ou lieux historiques, censés favoriser - ou manipuler - les émotions, susciter le recueillement ou la prise de recul.

Enfin, toutes les contributions nous renvoient au lien étroit que les lieux entretiennent avec l'identité collective. C'est manifeste dans la communication de Patrick Baudry qui constate que l'évolution de notre rapport au cimetière révèle une façon d'aborder différemment la mort et ce qu'elle implique dans l'organisation collective des vivants entre eux. Camille Mazé montre que c'est le rapport qu'une société entretient à son identité nationale qui suscite une éventuelle remise en question des "musées de la nation » au profit de projets visant à les européaniser ou à favoriser l'émergence de musées de l'Europe. La communication de Christine Pflüger nous rappelle en filigrane combien la réflexion critique sur le passé national-socialiste reste centrale dans l'identité allemande contemporaine. De son côté, Madeline Zielinksi explique comment la notion de " britannicité » est régulièrement réactivée en Grande-Bretagne lorsque le pays est en guerre, et elle démontre que la construction identitaire de la Grande-Bretagne au titre de nation guerrière a contribué à préparer le terrain de la réhabilitation des aviateurs du Bomber Command, finalement perçus comme des victimes de guerre, voire des héros. Raphäle Bertho montre pour sa part que la volonté de faire revivre le fastueux passé baroque de la ville de Dresde, même s'il est en partie imaginaire, renvoie finalement à l'identité à laquelle les habitants ont cherché à s'identifier en réaction à la perte des repères identitaires provoquée par les épreuves traversées aux cours des soixante-dix dernières années ; on pense au traumatisme de 1945 et à la dévastation provoqués par la Seconde Guerre mondiale, puis à la soviétisation contrainte de la société est-allemande, et enfin au choc du monde globalisé dans lequel la réunification l'a précipitée sans transition. Au fil de ces remous identitaires, le passé baroque a pu évoquer « la splendeur perdue » et renvoyer à une identité stable, valorisante et suffisamment consensuelle. 
L'histoire de ces lieux, leur effacement, résurgence ou surexposition dans la mémoire locale, nationale ou internationale, leur topographie muséale, esthétique ou didactique, leur dimension symbolique ou identitaire, toutes ces pistes sont autant d'entrées fructueuses dans la problématique multiforme qu'est la question de la construction des mémoires collectives.

Hélène Camarade EA 4593 CLARE Université Bordeaux Montaigne Institut Universitaire de France Hélène.Camarade@u-bordeaux-montaigne.fr 